\title{
Effects of Pilates on postural balance and functional autonomy of elderly: a randomized controlled trial
}

\author{
Laís Campos De Oliveira1,2, Deise Aparecida De Almeida Pires-Oliveira², Rafaela Claudia Alves Do Prado1, \\ Danielle Pereira D'avanso De Oliveira1', Tiago Del Antônio', Rodrigo Franco De Oliveira², Raphael Gonçalves De Oliveira',2
}

\begin{abstract}
Introduction: The Pilates method has become popular as a form of exercise, with good acceptance among the elderly, however, some variables need to be further explored, as the functional autonomy and postural balance. Objective: An objective of this study was to verify the effects of the Pilates method, based on the functional autonomy and postural balance in elderly women. Method: We recruited 24 volunteers who were randomly divided into two groups: experimental group (GE) who performed Pilates ( $n=12$ ); Control Group (CG) who performed exercise static stretching $(n=12)$. Both had a frequency of twice a week for eight weeks. The evaluations consisted of a battery of tests for functional autonomy of the elderly, the Group of Latin-American Development to the Maturity (GDLAM) protocol, six-minute walk test and static postural balance on a force platform. Results: The results showed significant results for GE in two tests of functional autonomy and the overall rate of functional autonomy $(p<0.05)$. Differences for the other tests were not found. Conclusions: The intervention protocol with Pilates, allowed the improvement of functional autonomy in elderly women, not having an effect on the six-minute walk test and the postural balance.
\end{abstract}

Keywords: Aged; Postural Balance; Exercise Movement Techniques.

Number in the Brazilian Registry of Clinical Trials: RBR-472p29

\section{INTRODUCTION}

The world population is aging; it is estimated that Brazil will have the sixth largest elderly population of the world, with 32 million people, in 2025. According to projections of the World Health Organization, the elderly population in Brazil will grow 16 times against five of the total population. IBGE data show that women still live longer than men. In 2060, the life expectancy of women will be 84.4 years, compared to 78 of men. (1)

The aging process may be understood as a set of structural and functional changes that occur in the body progressively. These changes contribute significantly to the loss of muscle strength, flexibility, endurance and cardiorespiratory capacity, impacting negatively on the functional autonomy of elderly. (2) This set of changes affect postural stability, resulting in decreased balance, the main risk factor for falls in the elderly. ${ }^{(3,4)}$

\section{METHOD}

The study is characterized as experimental, randomized and controlled. The sample consisted of 24 elderly women, aged between 60 and 75 years (mean 64,04 \pm 4,19 years),
One way to mitigate these problems is through physical exercise. ${ }^{(5)}$ A practice that has been increasingly sought by the elderly is the Pilates method. This method involves the use of springs as resistance resource; besides the own body weight, producing movements that require concentration and control of bodily structures. With the exercises of this method it is possible to gain benefits in relation to muscle strengthening, flexibility, endurance and postural stabilization, which are usually committed in the elderly, and interfere with the maintainability of the balance, providing positive effects on functional autonomy. ${ }^{(2,6,7)}$

The hypothesis is that a Pilates training designed to improve the elderly functionality can enable effective gains for components of functional autonomy and postural balance. Thus, this study aimed to verify the effect of Pilates on the functional autonomy and static postural balance of elderly.

living in the city of Jacarezinho, State of Parana, Brazil. The volunteers were randomly assigned to the control group (CG) and experimental group (GE), with 12 older each. The sample

Corresponding Author: Deise Aparecida de Almeida Pires-Oliveira. Address: Rua Marselha 591, Bairro Jardim Piza, Londrina (PR), Brasil. CEP: 86041-140.

Telephone:(43) 3371 7339/ 3371 9849. E-mail: deisepyres@gmail.com

${ }^{2}$ Centro de Ciências Biológicas e da Saúde, Universidade Norte do Paraná (UNOPAR), Londrina (PR), Brasil.

Full list of author information is available at the end of the article.

Financial support: There was no financial support for this study.

Submission date 10 June 2016; Acceptance date 25 August 2016; Publication online date 15 September 2016 
size was determined using the functional autonomy index presented by previous study as the basis of calculation. ${ }^{\left({ }^{8}\right)}$ By taking into consideration an error of $20 \%$ and $\alpha$ value of 0.05 , we identified the need to 12 participants in each group. All of them have signed the free, prior and informed consent. The study was approved by the Research Ethics Committee of the University Norte do Parana (protocol 513.001).

Inclusion criteria were: a) aged between 60 and 75 years; b) have independence to perform the activities of daily living (ADLs) and instrumental activities of daily living (IADLs); c) have the cognitive capacity to perform the exercises (score $>19$ on the Mini Mental State Examination) ${ }^{(9)} \mathrm{d}$ ) agree not to do any other exercise in addition to Pilates during the length of the research; e) did not perform systematic physical activity in the last 6 months. Exclusion criteria were: a) selfreported disorders in the neural or musculoskeletal system; b) presence of degenerative diseases; c) severe and chronic labyrinthitis; d) cardiovascular or respiratory diseases.

\section{Evaluation}

We performed the evaluation of functional autonomy using the tests of the Group of Latin-American Development to the Maturity (GDLAM) protocol, made up of: a) 10 meters walk $(10 \mathrm{~mW}) ; ;^{(10)}$ b) getting up from a seated position (GSP);(11) c) getting up from the ventral decubitus position (GVDP); ${ }^{(12)}$ d) getting up from a chair and movement around the house $\left.(\mathrm{GCMH}) ;{ }^{(13)} \mathrm{e}\right)$ putting on and taking off a shirt (PTS). ${ }^{(14)}$ Subjects performed two attempts for each test and the evaluator just recorded the fastest run (the shortest time). Further was calculated GDLAM Index (GI), through the following formula: $[(10 m W+G S P+G V D P+P T S) \times 2]+G C M H=/ 4$. The GI takes into consideration the execution of all tests, ${ }^{(15)}$ and is regarded as a general index of the elderly functional autonomy classification. Table 1 shows the scores for the weak, regular, good and very good levels in each test and in relation to GDLAM index (GI).

To evaluate the static postural balance (PB) we used a force platform, brand BIOMEC400 (EMG System of Brazil, Ltda, Sao Paulo). The evaluator explain the test procedures for the volunteer, who had a moment of familiarization with the equipment and the test that would be executed. Then the participants performed the task balance with bipedal support with open eyes. Three attempts of 60 seconds were performed, with equal rest time ( 60 seconds) between them. For further analysis of the data was used to average of attempts. The volunteers were barefoot, loose and relaxed arms alongside the body and the head positioned horizontally at ground level, being oriented to look at a fixed target (white paper circle with $3 \mathrm{~cm}$ in diameter), positioned in the wall at a distance of 2 meters and in eyes height.

Signs of strength of the ground reaction from the platform measurements were collected in a sample of $100 \mathrm{~Hz}$. All signs of strength went through a 2 th order low pass Butterworth filter at $35 \mathrm{~Hz}$. Then, signals were converted by a stabilographic analysis compiled with MatLab routines of the platform software (The Mathworks, Natick, MA). The balance parameters used were: displacement area of the center of pressure (CoP-A) expressed in square centimeters $\left(\mathrm{cm}^{2}\right)$ and the mean speed of oscillation expressed in centimeters per second $(\mathrm{cm} / \mathrm{s})$, in the anteroposterior (A/P) and mediolateral $(\mathrm{M} / \mathrm{L})$ directions, considering that these parameters showed good reliability in elderly. ${ }^{(16)}$

The six-minute walk test (6MWT) ${ }^{(17)}$ was performed in order to verify the cardiorespiratory fitness. The volunteers were in a minimum rest period of 10 minutes. During this period the contraindications were evaluated: a) blood pressure data; b) level of dyspnea (Borg Scale); c) respiratory and heart rate. The test was conducted in the hallway with a length of 30 meters without movement of people. The participants were trained previously and during the tests, encouraging phrases were used each minute through standardized phrases. At the end of each test, were annotated the number of given turns as well as the number of meters of the last lap in order to calculate the distance in meters $(\mathrm{m})$ covered by the voluntary. The test was performed twice, with an interval of 30 minutes between them in order to ensure the effect of learning. A chronometer and two cones were used in the tests.

All procedures were performed in the physical evaluation laboratory of the Health Sciences Center, at the State University of Norte do Paraná, by an evaluator-blinded regarding the study procedures and the allocation of the volunteers.

Table 1. Evaluation standard of the functional autonomy of the GDLAM protocol

\begin{tabular}{lcccccc}
\hline Tests/ Classification & $\begin{array}{c}10 \mathrm{~mW} \\
(\mathbf{s e c})\end{array}$ & $\begin{array}{c}\text { GSP } \\
\text { (sec.) }\end{array}$ & $\begin{array}{l}\text { GVDP } \\
\text { (sec.) }\end{array}$ & $\begin{array}{c}\text { GCMH } \\
\text { (sec.) }\end{array}$ & $\begin{array}{c}\text { PTS } \\
\text { (sec.) }\end{array}$ & $\begin{array}{c}\text { GI } \\
\text { (scores) }\end{array}$ \\
\hline Weak & $>7.09$ & $>11.19$ & $>4.40$ & $>43.00$ & $>13.14$ & $>27.42$ \\
Regular & $7.09-6.34$ & $11.19-9.55$ & $4.40-3.30$ & $43.00-38.69$ & $13.14-11.62$ & $27.42-24.98$ \\
Good & $6.33-5.71$ & $9.54-7.89$ & $3.29-2.63$ & $38.68-34.78$ & $11.61-10.14$ & $24.97-22.66$ \\
Very Good & $<5.71$ & $<7.89$ & $<2.63$ & $<34.78$ & $<10.14$ & $<22.66$ \\
\hline
\end{tabular}

Adapted from Dantas and Vale ${ }^{15}$; Vale et al. ${ }^{14}$ 


\section{Intervention}

\section{Experimental Group (GE)}

The intervention carried out by GE was 16 sessions of Pilates held twice a week for 8 weeks. The intervention lasted 60 minutes. Equipment used for the exercises were: Combo Chair, Cadillac Trapeze, Reformer Universal, Wall Unit, Half Moon, Step Barrel and Ladder Barrel (Instituto de Ortopedia e Fisioterapia, Sao Paulo, Brazil).

Were selected for this study 20 strengthening and stretching exercises to the main body segments (lower and upper limbs, trunk flexors and extensors). The sequence of exercises performed, the equipment used and the traditional name of each exercise of Pilates were respectively:

a) Initial stretches in Reformer Universal (Stretching the Chain Posterior, Front Splits, Mermaid);

b) Strengthening of the lower limbs in Universal Reformer (Foortwork) and Combo Chair (Foortwork Double Leg Pumps V Position, Pump One Leg Front, Going Up Front, Achilles Stretch);

c) Strengthening of trunk flexor and extensor muscles in Cadillac Trapeze (Sit Up, The Hundred, Teaser), Half Moon (Body Up and Down, Body Extension) and Step Barrel (Side sit Ups);

d) Strengthening of the upper limbs with $1 \mathrm{~kg}$ dumbbell (The Bug, Curls);

e) Final Stretches in the Wall Unit (Spine Stretch, Stretching the Spine) and Ladder Barrel (Stretch Back, Stretches Front).

All exercises were performed in a series of ten repetitions. The intensity of the springs was changed according to the evolution of the voluntary strength, keeping the number of series and repetitions. To determine the level of effort and consequently to changing loads, verbal descriptions were used according to the Borg scale CR10: (18) light load (Borg $\leq 2$ ), moderate load (Borg $>2-<5$ ), heavy load (Borg $\geq 5-<7$ ) and almost maximum load (Borg $\geq 7$ ). The level of effort maintained during the sessions was moderated (Borg between 3 and 4). Whenever the intensity of the exercise was changed the new load used was immediately noted in an individual form used for training log.

The exercises were selected attempting to improve muscle strength globally. The volunteers were instructed about the principles of the method (Center, Control, Concentration, Fluidity, Precision and Breath) that needed to be respected in the execution of each exercise.

The interventions were conducted by a professional certified in Pilates and experience with the method.

\section{Control Group (CG)}

The intervention carried out by CG was 16 static stretching sessions held twice a week for 8 weeks. The intervention lasted 60 minutes.

A sequence of twenty exercises was elaborated for the following regions of the body: cervical and upper limbs (upper trapezius, scalene, sternocleidomastoid, wrist and fingers extensors and flexors, deltoid, latissimus dorsi, triceps, pectoral, biceps, trunk flexors and extensors), side-chain and lower limbs stretching (oblique abdominal, hamstrings, quadriceps, adductors, glutes, abductors and triceps surae).

The exercises were performed actively with three series with a repetition maintained for 30 seconds, with equal rest time between series. This type of exercise followed the recommendations of the American College of Sports Medicine for adults, ${ }^{(19)}$ which establishes the benefits of stretching exercises to improve or maintain the flexibility when performed twice a week or more, held from 10 to 30 seconds in series that can vary from two to four.

\section{Statistical Analysis}

A descriptive data analysis was performed, expressed as mean and standard deviation. The verification of the data normality occurred by the Shapiro Wilk test. To check for differences between the groups regarding the initial physical characteristics (age, weight, height and BMI) and the preintervention assessments (functional autonomy, six-minute walk and postural balance) the U Mann Whitney test were used. The intra and inter-group comparisons at different times of the study were performed using variance analysis for repeated measures (ANOVA two way), followed by the t-test (LSD) which were used as post hoc when the variance analysis identified differences between groups. The percentage of change was calculated taking into account the absolute mean of each group pre and post-intervention. The admitted confidence interval for all tests was $95 \%(p<0,05)$. The data were processed in SPSS 20.0 (Chicago, IL, EUA) and BioEstat 5.3 (Belem, PA, Brazil) programs.

\section{RESULTS}

When analyzing the data for the initial physical characteristics (age, height, weight and BMI) and for physical tests applied pre-intervention, the U Mann-Whitney test found no significant differences among the groups, indicating that they were adequately randomized (Table 2 ).

In the intragroup comparison was identified significant differences in the PTS $(p=0,012), \operatorname{GCMH}(p=0,003)$ and GI $(0,007)$ functional tests the group that received the intervention with the Pilates method (GE). When performed comparison inter-groups (interaction between groups on time), the GVDP, PTS and GI functional tests showed a statistically significant difference $(p<0,05) n$ favor of the experimental 
group. The other tests showed no statistically significant differences intra or intergroup (Table 3).

\section{DISCUSSION}

The permanence of physical independence is impaired by the aging process, it is important to adopt strategies that can contribute to preserving the functional autonomy and

Table 2. Values of the initial characteristics of volunteers and test performed pre-intervention (baseline).

\begin{tabular}{lccc} 
& CG $(\mathbf{n}=\mathbf{1 2})$ & GE $(\mathbf{n}=12)$ & P \\
\cline { 2 - 3 } & Mean \pm SD & Mean \pm SD & \\
\hline Age (years) & $64.66 \pm 4.83$ & $63.41 \pm 3.55$ & 0.514 \\
Height (cm) & $159.50 \pm 0.05$ & $160.50 \pm 0.06$ & 0.266 \\
Weight (Kg) & $78.80 \pm 10.75$ & $67.66 \pm 6.86$ & 0.977 \\
BMI (Kg/m2) & $27.01 \pm 2.98$ & $28.36 \pm 3.46$ & 0.755 \\
10mW (s) & $7.86 \pm 1.85$ & $7.24 \pm 1.39$ & 0.443 \\
GSP (s) & $14.37 \pm 2.57$ & $16.36 \pm 6.37$ & 0.478 \\
GVDP (s) & $5.93 \pm 3.04$ & $4.55 \pm 1.62$ & 0.143 \\
PTS (s) & $12.12 \pm 5.87$ & $9.58 \pm 3.44$ & 0.291 \\
GCMH (s) & $22.44 \pm 2.89$ & $22.27 \pm 5.05$ & 0.887 \\
GI (scores) & $25.95 \pm 6.21$ & $24.83 \pm 5.81$ & 0.551 \\
6MWT (m) & $435 \pm 75.13$ & $460 \pm 60.17$ & 0.514 \\
CoP-A (cm ${ }^{2}$ ) & $1.19 \pm 0.67$ & $1 \pm 0.63$ & 0.242 \\
Mean speed of & & & \\
Oscillation A/P & $0.97 \pm 0.23$ & $0.94 \pm 0.15$ & 0.799 \\
(cm/s) & & & \\
Mean speed of & & $0.74 \pm 0.09$ & 0.755 \\
oscillation M/L & $0.79 \pm 0.26$ & & \\
(cm/s) & & & \\
\hline
\end{tabular}

quality of life of elderly. This preservation can be obtained through the practice of physical exercises, especially when they are conducted regularly and systematically, which is the main factor for the maintenance of strength, endurance, cardiorespiratory fitness, coordination and balance, preventing falls and improving the quality of life of the elderly population. ${ }^{(2,5,8)}$

One way to evaluate the functional autonomy of elderly is through GDLAM protocol, which was developed specifically for the elderly, consisting of activities of daily living measures, such as: walking, getting up and dressing. ${ }^{(14,15)}$ Rodrigues et al. ${ }^{(8)}$ utilized the GDLAM protocol to evaluate the functional autonomy of elderly submitted to Pilates method. The experimental group follows the same protocol of this study (Pilates twice a week for eight weeks). As in this study, the authors observed significant differences for three tests, including the General Index of functional autonomy.

In Gl, the elderly of CG increased from an average of 25,95 (Regular) to 23,24 (Good), while the elderly from the GE presented 24,83 (Good) in pre-intervention and 18,65 (Very Good) in post-intervention evaluation. The experimental group showed a significant difference to the IG in both intergroup $(p=0,040)$ and intra-group comparison $(p=0,007)$.

Other studies have investigated the functional autonomy of elderly and made use of different assessment protocols. ${ }^{(20-23)}$ Fourie et al. ${ }^{(20)}$ observed that eight weeks of Pilates performed three times a week enabled significant improvement only in the group that performed exercises in functional tests of upper and lower limbs. With the same period of intervention and frequency, Gildenhuys et al. ${ }^{(21)}$ and Irez et al. ${ }^{(22)}$ also verified the effects of Pilates to a battery of functional tests. Plachy, Kovách and Bognár ${ }^{(23)}$ found that Pilates associated or not

Table 3. Comparison intra-group of the performed evaluations pre and post-intervention and intergroup interaction over time (ANOVA two way).

\begin{tabular}{|c|c|c|c|c|c|c|c|}
\hline & \multicolumn{3}{|c|}{ CG $(n=12)$} & \multicolumn{3}{|c|}{ GE $(n=12)$} & \multirow{3}{*}{$\begin{array}{c}\text { Interaction } \\
\text { between groups } \\
\text { on time (P) }\end{array}$} \\
\hline & Pre-intervention & Post-intervention & \multirow{2}{*}{ Change (\%) } & Pre-intervention & Post-intervention & \multirow{2}{*}{ Change (\%) } & \\
\hline & Mean \pm SD & Mean \pm SD & & Mean \pm SD & Mean \pm SD & & \\
\hline $10 \mathrm{~mW}(\mathrm{~s})$ & $7.86 \pm 1.85$ & $6.79 \pm 1.52$ & -13.61 & $7.24 \pm 1.39$ & $6.35 \pm 1.06$ & -12.29 & 0.373 \\
\hline GSP (s) & $14.37 \pm 2.57$ & $13.54 \pm 3.46$ & -5.78 & $16.36 \pm 6.37$ & $13.37 \pm 3.05$ & -18.28 & 0.358 \\
\hline GVDP (s) & $5.93 \pm 3.04$ & $5.04 \pm 2.39$ & -14.84 & $4.55 \pm 1.62$ & $2.96 \pm 0.48$ & -35.09 & 0.013 \\
\hline PTS (s) & $12.12 \pm 5.87$ & $10.42 \pm 3.74$ & -14.10 & $9.58 \pm 3.44$ & $5.52 \pm 1.98^{*}$ & -42.34 & 0.001 \\
\hline GCMH (s) & $22.44 \pm 2.89$ & $20.79 \pm 4.33$ & -7.310 & $22.27 \pm 5.05$ & $17.88 \pm 2.03^{*}$ & -19.75 & 0.053 \\
\hline GI (scores) & $25.95 \pm 6.21$ & $23.24 \pm 5.29$ & -10.48 & $24.83 \pm 5.81$ & $18.65 \pm 2.92^{*}$ & -24.89 & 0.040 \\
\hline 6MWT (m) & $435 \pm 75.13$ & $462.5 \pm 78.29$ & 6.32 & $460 \pm 60.17$ & $487.5 \pm 49.74$ & 5.98 & 0.152 \\
\hline CoP-A $\left(\mathrm{cm}^{2}\right)$ & $1.19 \pm 0.67$ & $1.18 \pm 0.65$ & -0.84 & $1 \pm 0.63$ & $0.95 \pm 0.49$ & -5.94 & 0.146 \\
\hline $\begin{array}{l}\text { Mean Speed A/P } \\
(\mathrm{cm} / \mathrm{s})\end{array}$ & $0.97 \pm 0.23$ & $0.96 \pm 0.22$ & -2.04 & $0.94 \pm 0.15$ & $1.04 \pm 0.49$ & 9.47 & 0.178 \\
\hline $\begin{array}{l}\text { Mean Speed M/L } \\
(\mathrm{cm} / \mathrm{s})\end{array}$ & $0.79 \pm 0.26$ & $0.73 \pm 0.11$ & -8.75 & $0.74 \pm 0.09$ & $0.71 \pm 0.07$ & -4.00 & 0.467 \\
\hline
\end{tabular}

Legend: * pre and post-intervention intra-group difference $(p<0,05)$. 
with water aerobics, promote improvement of the functional autonomy of elderly.

The six-minute walk test has also been used to assess the functional capacity. This test can also predict cardiorespiratory fitness condition and is an overall indicator of the responses of all the systems involved during exercise. ${ }^{(24)}$ The appropriate level of this fitness is required to perform the activities of daily living; the loss of this fitness could reduce the individual's ability to live independently. ${ }^{(25)}$

The distance walked by the volunteers of this study did not change between pre and post-intervention evaluations (intragroup comparison), as well as there were no differences between groups over time (intergroup comparison). Gildenhuys et al. (21) evaluated the cardiorespiratory capacity through the six-minute walk test in elderly submitted to the Pilates method and found that eight weeks of intervention did not allow changes of this variable, corroborating with the present study. However, Plachy, Kovách and Bognár ${ }^{(23)}$ verified significant improvements to the six-minute walk test in elderly practitioners of Pilates. Nevertheless, differently from the protocols adopted in most studies, ${ }^{(8,20-22)}$ the authors used three sessions per week over 24 weeks, which may explain the observed improvement.

Regarding the postural balance evaluated through the force platform, this study showed no significant results of pre and post-intervention of the Pilates method, so finding no evidence that 16 technical sessions are sufficient to improve the static balance of elderly in bipedal support. But studies conducted with elderly have shown that regular physical activity and the Pilates technique can benefit the postural balance. ${ }^{(8,22)}$

A study ${ }^{(3)}$ showed the effects of Pilates after eight weeks of intervention and found that the dynamic balance of the elderly changed significantly contributing to decreased risk of falls. Similarly, another study found improvement in dynamic balance and consequent reduction in the risk of falls in elderly subjected to 12 weeks of intervention. ${ }^{(22)}$

Bird et al. ${ }^{(26)}$ evaluated the static and dynamic balance in elderly women with eight weeks of Pilates intervention, verifying that the variables improved during the study, but showed no significance. In the present study was performed only the static balance variable which also obtained an improvement in values but without statistical significance $(p<0,05)$.

Once the Pilates technique began to be investigated with higher intensity just recently, other studies must be performed in order to test different intervention, intensity, frequency and training time protocols. Was expected that this method would enable improvement in postural balance but it did not occur. Thus, it is suggested for further studies that should be tested different positions of the lower limbs on the force platform, such as tandem, semi-tandem and unipodal. The test can also be performed without the aid of the visual system, increasing the challenge about the evaluated.

\section{CONCLUSION}

Pilates exercise program carried out for eight weeks, twice a week, led to an improvement of functional autonomy in elderly women. Significant changes were not found in the six-minute walk test and static postural balance.

\section{AUTHOR'S CONTRIBUTIONS:}

LCO: contributed with the preparation of the study design and development, acquisition and analysis of data, and approve the final version.

DAAPO: contributed with the preparation of the study design and development, acquisition and analysis of data, review critically the final content, and approve the final version.

RCAP: contributed with the development of the study, data acquisition, and approve the final version.

DPDO: contributed with the development of the study, data acquisition, and approve the final version.

TDO: contributed with data acquisition, review critically the final content, and approve the final version.

RFO: contributed with the preparation of the study design and development, acquisition and analysis of data, review critically the final content, and approve the final version.

RGO: contributed with the preparation of the study design and development, acquisition and analysis of data, and approve the final version.

\section{CONFLICTS OF INTEREST:}

The authors declare that they have no conflicts of interest in the research.

\section{AUTHOR DETAILS}

${ }^{1}$ Centro de Ciências da Saúde, Universidade Estadual do Norte do Paraná (UENP), Jacarezinho (PR), Brasil.

\section{REFERENCES}

1. Instituto Brasileiro de Geografia e Estatística. Idosos no mundo. Internet site address: <http://www.ibge.gov.br.> acesso em: 01/10/2014

2. Pérez VSC, Haas AN, Wolff SS. Analysis of activities in the daily live of older adults exposed to the Pilates Method. J Bodyw Mov Ther.2013;18(3):32631.

3. Pata RW, Lord K, Lamb J. The effect of Pilates based exercise on mobility, postural stability, and balance in order to decrease fall risk in older adults. J Bodyw Mov Ther. 2013;18(3):361-7.

4. Hyun J, Hwangbo K, Lee C. The effects of Pilates Mat exercise on the Balance Ability of elderly females. J Phys Ther Sci. 2013;26(2):291-3.

5. Geraldo LVOB, Moraes AMN, Fonseca HR, Emerick RF, Deslandes SEAC. Relationship between level of Independence in activities of daily living and estimated cardiovascular capacity in elderly women. Arch Gerontol Geriatr. 2014;59(2):367-71.

6. Di Lorenzo. Pilates: what is it? Should it be used in rehabilition. Sports Health. 2011;3(4):352-61.

7. Shea S, Moriello G. Feasibility and outcomes of a classical Pilates program on lower extremity strength, posture, balance, gait, and quality of life in someone with impartiments due to a stroke. J Bodyw Mov Ther. 2013;18(3):332-60.

8. Rodrigues BGS, Cader AS, Torres NVOB, Oliveira EM, Dantas EHM. Pilates method in personal autonomy, static balance and quality of life of elderly females. J Bodyw Mov Ther. 2010;14(2):195-202.

9. Hughes MA, Duncan PW, Rose DK, Chandler JM, Studenski SA. The relationship of postural sway to sensoriomotor function, functional performance, and disability in the elderly. Arch Phys Med Rehabil. $1996 ; 77(6): 567-72$

10. Sipila S, Multanen J, Kallinen M, Era P, Suominen H. Effects of strength and endurance training on isometric muscle strenght and walking speed in elderly women. Acta Physiol Scand. 1996;156(4)457-64. 
11. Guralnik JM, Simonsick EM, Ferucci L, Glynn RJ, Robert J, Berk-man LF, et al. A short physical performance battery assessing lower extremity function: association with self-reported disability and prediction of mortality and nursing home admission. J Gerontol. 1994;49(2):85-94.

12. Alexander nb, Ulbrich J, Raheja A, Channer D. Rising from the floors in older adults. J Am Geriatr Soc. 1997;45(5):564-69.

13. Andreotti R, Okuma SS. Validação de uma bateria de testes de atividades da vida diária para idosos fisicamente independentes. Rev Paul Educ Fis. 1999;13(1):46-66.

14. Vale RGS, Pernambuco CS, Novaes JS, Dantas EHM. Teste de autonomia funcional: vestir e tirar uma camiseta (VTC). Rev Bras Cienc Mov. 2006;14(3):71-8

15. Dantas EHM, Vale RGS, Pernambuco CS. Protocolo GDLAM de avaliação da autonomia funcional. Fit Perform J. 2004;3(3):175-83.

16. Silva RA, Bilodeau M, Parreira RB, Teixeira DC, Amorim CF. Age-related differences in time-limit performance and force platform-based balance measures during one-leg stance. J Electromyogr Kinesiol. 2013;23(3):634-9.

17. Jones CJ, Rikli RE. Senior Fitness Test Manual. Champaign, IL: Human Kinetics; 2001.

18. Borg GAV. Psychophysical bases of perceived exertion. Med Sci Sports Exerc. 1982;14(5):377-81.
19. Garber CE, Blissmer B, Deschenes MR, Franklin BA, Lamonte MJ, Lee IM. et al. American College of Sports Medicine position stand. Quantity and quality of exercise for developing and maintaining cardiorespiratory, musculoskeletal, and neuromotor fitness in apparently healthy adults: guidance for prescribing exercise. Med Sci Sports Exerc. 2011;43(7):1334-59.

20. Fourie M, Gildenhuys GM, Shaw I, Shaw BS, Toriola AL, Goon DT. Effects of a mat Pilates programme on muscular strength and endurance in elderly women. Afr J Phys Health Education Recreation Dance. 2012;18(2):299-307.

21. Gildenhuys GM, Fourie M, Shaw I, Shaw BS, Toriola AL, Witthuhn J. Evaluation of Pilates training on agility, functional mobility and cardiorespiratory fitness in elderly women. Afr J Phys Health Education Recreation Dance. 2013;19(2):505-12.

22. Irez GB, Ozdemir RA, Evin R, Irez SG, Korkusuz F. Integrating Pilates exercise into an exercise program for $65+$ year-old women to reduce falls. J Sports Sci Med. 2011;10(1):105-11.

23. Plachy JK, Kovách MV, Bognár J. Improving flexibility and endurance of elderly women through a six-month training programme. Hum Mov. 2012;13(1):p.22-7.

24. Timson, BF, Falls HB, Wilson TE, Zimmerman SD. Effect of muscle strength on $\mathrm{VO}_{2}$ plateau occurrence rate. Isokinet Exerc Sci. 2008;16(4):231-7.

25. Milton D, Porcari JP, Foster C, Gibson M, Undermann B, Greany J, et al. The effect of functional exercise training of functional fitness levels of older adults. Gundersen Lutheran Med J. 2008;5(1):1-5.

26. Bird ML, Hill KD, Fell JW. A randomized controlled study investigating static and dynamic balance in older adults after training with pilates. Arch Phys Med Rehabil. 2012;93(1):43-9. 